https://helda.helsinki.fi

\title{
Two weight inequalities for bilinear forms
}

\section{$\mathrm{Li}$, Kangwei}

2017-01

$\mathrm{Li}, \mathrm{K} 2017$, ' Two weight inequalities for bilinear forms ' , Collectanea Mathematica , vol. 68 , no. 1 , pp. 129-144 . https://doi.org/10.1007/s13348-016-0182-2

http://hdl.handle.net/10138/307531

https://doi.org/10.1007/s13348-016-0182-2

other

acceptedVersion

Downloaded from Helda, University of Helsinki institutional repository.

This is an electronic reprint of the original article.

This reprint may differ from the original in pagination and typographic detail.

Please cite the original version. 


\title{
TWO WEIGHT INEQUALITIES FOR BILINEAR FORMS
}

\author{
KANGWEI LI
}

\begin{abstract}
Let $1 \leq p_{0}<p, q<q_{0} \leq \infty$. Given a pair of weights $(w, \sigma)$ and a sparse family $\mathcal{S}$, we study the two weight inequality for the following bi-sublinear form

$$
B(f, g)=\sum_{Q \in \mathcal{S}}\left\langle|f|^{p_{0}}\right\rangle_{Q}^{\frac{1}{p_{0}}}\left\langle|g|^{q_{0}^{\prime}}\right\rangle_{Q}^{\frac{1}{q_{0}^{\prime}}} \lambda_{Q} \leq \mathcal{N}\|f\|_{L^{p}(w)}\|g\|_{L^{q^{\prime}}(\sigma)} .
$$

When $\lambda_{Q}=|Q|$ and $p=q$, Bernicot, Frey and Petermichl showed that $B(f, g)$ dominates $\langle T f, g\rangle$ for a large class of singular non-kernel operators. We give a characterization for the above inequality and then obtain the mixed $A_{p}-A_{\infty}$ estimates and the corresponding entropy bounds when $\lambda_{Q}=|Q|$ and $p=q$. We also proposed a new conjecture which implies both the one supremum conjecture and the separated bump conjecture.
\end{abstract}

\section{IntRoduction AND MAIN RESUlts}

The weighted theory for Calderón-Zygmund operators has achieved several advances in the last decades. The quantitative relation between the weighted bound of the operator and the $A_{p}$ characteristics has attracted many authors' interest. The climax of this topic is the settle of the $A_{2}$ conjecture, which was due to Hytönen [9]. Lerner [17] also gave a simple proof for it by reducing the problem to study the so-called sparse operators. We refer the readers to $[9,17]$ and the reference therein for an overview of this topic.

Later on, Hytönen and Lacey [11] extended the $A_{2}$ theorem to the mixed $A_{p}-A_{\infty}$ estimate, and proposed the famous one supremum conjecture (which will be recalled below), which is in the theme of "finding the minimal sufficient condition such that the two weight inequality holds". As far as we know, this conjecture is still open. Another problem in the same theme is the so-called separated bump conjecture (which will be recalled in Section 5), which arised from work of Cruz-Uribe and Pérez [4, 5], and Cruz-Uribe and Reznikov and Volberg [6]. The separated bump conjecture was just

Date: August 21, 2018.

2010 Mathematics Subject Classification. 42B25.

Key words and phrases. $A_{p}-A_{\infty}$ estimates, two weight theorem, one supremum estimate, separated bump conjecture.

The author is supported by the European Union through Tuomas Hytönen's ERC Starting Grant "Analytic-probabilistic methods for borderline singular integrals". He is a member of the Finnish Centre of Excellence in Analysis and Dynamics Research. 
verified for the log-bumps [6, 1]. In general, it is still unknown, see also in [14] for more details.

Recently, Bernicot, Frey and Petermichl [2] studied the weighted theory beyond Calderón-Zygmund theory. They gave the sharp weighted estimates for a large class of singular non-kernel operators by proving a domination theorem. To be precise, they showed that, if $T$ is bounded on $L^{2}$ and satisfies some cancellation property and Cotlar type inequality, then for $f, g$ supported in $5 Q_{0}$ for some cube $Q_{0}$, there exists some sparse family $\mathcal{S}_{0}$ such that

$$
\left|\int_{Q_{0}} T f \cdot g d \mu\right| \leq C \sum_{P \in \mathcal{S}_{0}} \mu(P)\left\langle|f|^{p_{0}}\right\rangle_{5 P}^{1 / p_{0}}\left\langle|g|^{q_{0}^{\prime}}\right\rangle_{5 P}^{1 / q_{0}^{\prime}}
$$

where

$$
\langle h\rangle_{Q}=\frac{1}{\mu(Q)} \int_{Q} h(x) d \mu,
$$

and $1 \leq p_{0}<q_{0} \leq \infty$. Recall that we say $\mathcal{S}$ is sparse if for any $Q \in \mathcal{S}$,

$$
\mu\left(\bigcup_{Q^{\prime} \in \mathcal{S}, Q^{\prime} \subsetneq Q} Q^{\prime}\right) \leq \frac{1}{2} \mu(Q) .
$$

The above result is in the setting of locally compact separable metric space equipped with a doubling Borel measure $\mu$, finite on compact sets and strictly positive on any nonempty open set. In the following, we simply use $|Q|$ stands for $\mu(Q)$.

It is well-known that there exists a finite collection of adjacent dyadic systems $\mathcal{D}_{k}, k=1, \cdots, K$, such that for any cube $Q$, there exists some $1 \leq k_{0} \leq K$ such that $5 Q \subset \tilde{Q} \in \mathcal{D}_{k_{0}}$ and $|\tilde{Q}| \approx|Q|$ (e.g. see [10]). Then RHS(1.1) can be dominated by

$$
\int_{\mathbb{R}^{n}} \sum_{k=1}^{K} \sum_{Q \in \mathcal{D}_{k}}\left\langle|f|^{p_{0}}\right\rangle_{Q}^{1 / p_{0}}\left\langle|g|^{q_{0}^{\prime}}\right\rangle_{Q}^{1 / q_{0}^{\prime}} \sum_{\substack{P \in \mathcal{S}_{0} \\ \tilde{P}=Q}} \mathbf{1}_{P}(x) d x .
$$

Then following similar arguments as that in [7], it suffices to consider the following bi-sublinear form

$$
B(f, g)=\sum_{Q \in \mathcal{S}}\left\langle|f|^{p_{0}}\right\rangle_{Q}^{\frac{1}{p_{0}}}\left\langle|g|^{q_{0}^{\prime}}\right\rangle_{Q}^{\frac{1}{q_{0}^{\prime}}} \lambda_{Q}
$$

where $\mathcal{S}$ is a sparse family (here we generalize $|Q|$ to general sequence $\lambda_{Q}$ ). This is the main object in this paper.

Our first result concern the characterization of two weight norm inequality for the bilinear form.

Theorem 1.2. Let $(w, \sigma)$ be a pair of weights and $p_{0}<p, q<q_{0}$. Suppose that $\mathcal{N}$ is the best constant such that the following two weight inequality holds

$$
B(f, g) \leq \mathcal{N}\|f\|_{L^{p}(w)}\|g\|_{L^{q^{\prime}(\sigma)}}
$$


Denote

$$
\tau_{Q}=\langle u\rangle_{Q}^{-\frac{1}{p_{0}^{\prime}}}\langle v\rangle_{Q}^{-\frac{1}{q_{0}}} \frac{\lambda_{Q}}{|Q|}, u:=w^{\frac{p_{0}}{p_{0}-p}}, v:=\sigma^{\frac{q_{0}^{\prime}}{q_{0}^{\prime}-q^{\prime}}}
$$

and

$$
T_{F}(f)=\sum_{\substack{Q \in \mathcal{S} \\ Q \subset F}} \tau_{Q}\langle f\rangle_{Q} \mathbf{1}_{Q}
$$

Then we have

$$
\mathcal{N} \approx \mathfrak{T}_{s}+\mathfrak{T}_{s}^{*}
$$

where $s \in(1, \infty]$ is determined by

$$
\frac{1}{s}=\left(\frac{1}{q}-\frac{1}{p}\right)_{+}:=\max \left\{\frac{1}{q}-\frac{1}{p}, 0\right\}
$$

and

$$
\begin{aligned}
& \mathfrak{T}_{s}:=\sup _{\mathcal{F}}\left\|\left\{\frac{\left\|T_{F}(u)\right\|_{L^{q}(v)}}{u(F)^{1 / p}}\right\}_{F \in \mathcal{F}}\right\|_{\ell^{s}}, \\
& \mathfrak{T}_{s}^{*}:=\sup _{\mathcal{G}}\left\|\left\{\frac{\left\|T_{G}(v)\right\|_{L^{p^{\prime}}(u)}}{v(G)^{1 / q^{\prime}}}\right\}_{G \in \mathcal{G}}\right\|_{\ell^{s}},
\end{aligned}
$$

and the supremums are taken over all subcollections $\mathcal{F}$ and $\mathcal{G}$ of $\mathcal{S}$ that are sparse with respect to $u$ and $v$, respectively.

For the case of $p_{0}=1, q_{0}=\infty$, this result is already known in [15] for $p \leq q$ and [21] for $p>q$, see also in [8] for an unified approach for both cases. In this sense, our result extends from the special case $p_{0}=1, q_{0}=\infty$ to general cases. Next, we are concerned with the mixed $A_{p}-A_{\infty}$ type estimate.

Theorem 1.6. Let $(w, \sigma)$ be a pair of weights, $\lambda_{Q}=|Q|, p=q$ and $\mathcal{N}$ be the best constant such that (1.3) holds. Then

$$
\mathcal{N} \lesssim[v]_{A_{r}}^{\frac{1}{q_{0}^{\prime}}-\frac{1}{p^{\prime}}}\left([u]_{A_{\infty}}^{\frac{1}{p}}+[v]_{A_{\infty}}^{\frac{1}{p^{\prime}}}\right)
$$

where

$$
r=\left(\frac{q_{0}}{p}\right)^{\prime}\left(\frac{p}{p_{0}}-1\right)+1 .
$$

We point out Theorem 1.6 improves the main result in [2]. Indeed, notice that Bernicot, Frey and Petermichl showed that, if $\sigma=w^{1-p^{\prime}}$, i.e., $u=v^{1-r^{\prime}}$, then

Since

$$
\mathcal{N} \lesssim[v]_{A_{r}}^{\max \left\{\frac{1}{q_{0}^{\prime}}, \frac{1}{p_{0}(r-1)}\right\}}
$$

we have

$$
[u]_{A_{\infty}}^{\frac{1}{p}} \leq[u]_{A_{r^{\prime}}}^{\frac{1}{p}}=[v]_{A_{r}}^{\frac{1}{p(r-1)}} \text {, and }[v]_{A_{\infty}}^{\frac{1}{p^{\prime}}} \leq[v]_{A_{r}}^{\frac{1}{p^{\prime}}}
$$

$$
[v]_{A_{r}}^{\frac{1}{q^{\prime}}-\frac{1}{p^{\prime}}}\left([u]_{A_{\infty}}^{\frac{1}{p}}+[v]_{A_{\infty}}^{\frac{1}{p^{\prime}}}\right) \leq[v]_{A_{r}}^{\frac{1}{p_{0}(r-1)}}+[v]_{A_{r}}^{\frac{1}{q^{\prime}}} \leq 2[v]_{A_{r}}^{\max \left\{\frac{1}{q_{0}^{\prime}}, \frac{1}{p_{0}(r-1)}\right\}} .
$$


Finally we study the one supremum estimate. In [11], Hytönen and Lacey proposed the following one supremum conjecture, which is an extension of the $A_{p}-A_{\infty}$ estimate.

Conjecture 1.7. Let $T$ be a Calderón-Zygmund operator, $w, \sigma$ be weights and $1<p<\infty$. Then

$$
\|T(\cdot \sigma)\|_{L^{p}(\sigma) \rightarrow L^{p}(w)} \lesssim \sup _{Q}\langle w\rangle_{Q}^{\frac{1}{p}}\langle\sigma\rangle_{Q}^{\frac{1}{p^{\prime}}}\left(A_{\infty}(w, Q)^{\frac{1}{p^{\prime}}}+A_{\infty}(\sigma, Q)^{\frac{1}{p}}\right),
$$

where for any weight $v$,

$$
A_{\infty}(v, Q):=\frac{1}{v(Q)} \int_{Q} M\left(v \chi_{Q}\right)(x) d x .
$$

Here we give some partial answer to this conjecture for the general indices $1 \leq p_{0}<q_{0} \leq \infty$. Define

$$
A_{r}(v, u, Q)=\langle v\rangle_{Q}^{\frac{1}{p}-\frac{1}{q_{0}}}\langle u\rangle_{Q}^{\left(\frac{1}{p}-\frac{1}{q_{0}}\right)(r-1)},
$$

and

$$
\begin{aligned}
{[v, u]_{\left(A_{r}\right)\left(\phi\left(A_{\infty}\right)\right)}:=\sup _{Q} A_{r}(v, u, Q)\left(A_{\infty}(v, Q)\right)^{\frac{1}{p^{\prime}}} \phi\left(A_{\infty}(v, Q)\right) ; } \\
{[u, v]_{\left(A_{r^{\prime}}\right)\left(\psi\left(A_{\infty}\right)\right)}:=\sup _{Q} A_{r^{\prime}}(u, v, Q)\left(A_{\infty}(u, Q)\right)^{\frac{1}{p}} \psi\left(A_{\infty}(u, Q)\right) . }
\end{aligned}
$$

We have the following one supremum estimate

Theorem 1.8. Let $(w, \sigma)$ be a pair of weights, $\lambda_{Q}=|Q|, p=q$ and $\mathcal{N}$ be the best constant such that (1.3) holds. Let $\phi, \psi$ be increasing functions such that

Then

$$
\int_{1 / 2}^{\infty}\left(\frac{1}{\phi(t)}+\frac{1}{\psi(t)}\right) \frac{d t}{t}<\infty
$$

$$
\mathcal{N} \lesssim[v, u]_{\left(A_{r}\right)\left(\phi\left(A_{\infty}\right)\right)}+[u, v]_{\left(A_{r^{\prime}}\right)\left(\psi\left(A_{\infty}\right)\right)},
$$

For the case of $p_{0}=1$ and $q_{0}=\infty$, this was shown by Lacey and Spencer in [16], which was referred to as the separated entropy bounds. Now consider another definition of $A_{\infty}$ weights, namely,

$$
[w]_{A_{\infty}}^{\exp }:=\sup _{Q}\langle w\rangle_{Q} \exp \left(\left\langle\log w^{-1}\right\rangle_{Q}\right)
$$

It is showed in [13] that

$$
[w]_{A_{\infty}} \leq c_{n}[w]_{A_{\infty}}^{\exp }
$$

We shall show that, if we replace $A_{\infty}$ with $A_{\infty}^{\exp }$, then we can relax the decay condition of $\phi$ and $\psi$ slightly. To be precise, define

$$
A_{\infty}^{\exp }(v, Q):=\langle v\rangle_{Q} \exp \left(\left\langle\log v^{-1}\right\rangle_{Q}\right)
$$

and

$$
[v, u]_{\left(A_{r}\right)\left(\Phi\left(A_{\infty}^{\exp }\right)\right)}:=\sup _{Q} A_{r}(v, u, Q)\left(A_{\infty}^{\exp }(v, Q)\right)^{\frac{1}{p^{\prime}}} \Phi\left(A_{\infty}^{\exp }(v, Q)\right)
$$




$$
[u, v]_{\left(A_{r^{\prime}}\right)\left(\Psi\left(A_{\infty}^{\exp }\right)\right)}:=\sup _{Q} A_{r^{\prime}}(u, v, Q)\left(A_{\infty}^{\exp }(u, Q)\right)^{\frac{1}{p}} \Psi\left(A_{\infty}^{\exp }(u, Q)\right) .
$$

We have the following result

Theorem 1.9. Let $(w, \sigma)$ be a pair of weights, $\lambda_{Q}=|Q|, p=q$ and $\mathcal{N}$ be the best constant such that (1.3) holds. Let $\Phi, \Psi$ be increasing functions such that

$$
\int_{1 / 2}^{\infty}\left(\frac{1}{\Phi(t)^{p}}+\frac{1}{\Psi(t)^{p^{\prime}}}\right) \frac{d t}{t}<\infty
$$

Then

$$
\mathcal{N} \lesssim[v, u]_{\left(A_{r}\right)\left(\Phi\left(A_{\infty}^{\exp }\right)\right)}+[u, v]_{\left(A_{r^{\prime}}\right)\left(\psi\left(A_{\infty}^{\exp }\right)\right)},
$$

The organization of the paper will be as follows. In Section 2, we present the proof of Theorem 1.2. In section 3, we prove Theorem 1.6. We study the one supremum estimate in Section 4, which contains the proof of Theorems 1.8 and 1.9. And we end this manuscript with a generalized question in Section 5 .

\section{A CHARACTERIZATION OF THE TWO WEIGHT NORM INEQUALitiES FOR BILINEAR FORMS}

In this section, we give a proof for Theorem 1.2. Recall that $u=w^{\frac{p_{0}}{p_{0}-p}}$ and $v=\sigma^{\frac{q_{0}^{\prime}}{q_{0}^{\prime}-q^{\prime}}}$. We can write the two weight inequality as follows

$$
B(f, g)=\sum_{Q \in \mathcal{S}}\left\langle|f|^{p_{0}}\right\rangle_{Q}^{\frac{1}{p_{0}}}\left\langle|g|^{q_{0}^{\prime}}\right\rangle_{Q}^{\frac{1}{q_{0}^{\prime}}} \lambda_{Q} \leq C\left\||f|^{p_{0}} u^{-1}\right\|_{L^{p / p_{0}(u)}}^{1 / p_{0}}\left\||g|^{q_{0}^{\prime}} v^{-1}\right\|_{L^{q^{\prime} / q_{0}^{\prime}(v)}}^{1 / q^{\prime}},
$$

which is equivalent to

$$
\sum_{Q \in \mathcal{S}}\left(\left\langle|f|^{p_{0}}\right\rangle_{Q}^{u}\right)^{\frac{1}{p_{0}}}\left(\left\langle|g|^{q_{0}^{\prime}}\right\rangle_{Q}^{v}\right)^{\frac{1}{q_{0}^{\prime}}} \lambda_{Q}\langle u\rangle_{Q}^{\frac{1}{p_{0}}}\langle v\rangle_{Q}^{\frac{1}{q_{0}^{\prime}}} \leq C\left\||f|^{p_{0}}\right\|_{L^{p / p_{0}(u)}}^{1 / p_{0}}\left\||g|^{q_{0}^{\prime}}\right\|_{L^{q^{\prime} / q_{0}^{\prime}(v)}}^{1 / q_{0}^{\prime}}
$$

where for any function $h$ and weight $w$,

$$
\langle h\rangle_{Q}^{w}:=\frac{1}{w(Q)} \int_{Q} h \mathrm{~d} w .
$$

Follow the spirit in [12], we claim that (2.1) is equivalent to

$$
\sum_{Q \in \mathcal{S}}\langle f\rangle_{Q}^{u}\langle g\rangle_{Q}^{v} \lambda_{Q}\langle u\rangle_{Q}^{\frac{1}{p_{0}}}\langle v\rangle_{Q}^{\frac{1}{q_{0}^{\prime}}} \leq C\|f\|_{L^{p}(u)}\|g\|_{L^{q^{\prime}}(v)}
$$

In fact, if (2.1) holds, then (2.2) follows immediately from Hölder's inequality. On the other hand, if (2.2) holds, then we have

$$
\sum_{Q \in \mathcal{S}}\left(\left\langle|f|^{p_{0}}\right\rangle_{Q}^{u}\right)^{\frac{1}{p_{0}}}\left(\left\langle|g|^{q_{0}^{\prime}}\right\rangle_{Q}^{v}\right)^{\frac{1}{q_{0}^{\prime}}} \lambda_{Q}\langle u\rangle_{Q}^{\frac{1}{p_{0}}}\langle v\rangle_{Q}^{\frac{1}{q_{0}^{\prime}}}
$$




$$
\begin{aligned}
& \leq \sum_{Q \in \mathcal{S}}\left\langle M_{p_{0}, u}^{\mathcal{S}}(f)\right\rangle_{Q}^{u}\left\langle M_{q_{0}^{\prime}, v}^{\mathcal{S}}(g)\right\rangle_{Q}^{v} \lambda_{Q}\langle u\rangle_{Q}^{\frac{1}{p_{0}}}\langle v\rangle_{Q}^{\frac{1}{q_{0}^{\prime}}} \\
& \leq C\left\|M_{p_{0}, u}^{\mathcal{S}}(f)\right\|_{L^{p}(u)}\left\|M_{q_{0}^{\prime}, v}^{\mathcal{S}}(g)\right\|_{L^{q^{\prime}}(v)} \\
& \leq C_{p_{0}, p, q_{0}, n} C\|f\|_{L^{p}(u)}\|g\|_{L^{q^{\prime}}(v)},
\end{aligned}
$$

where

$$
M_{p, u}^{\mathcal{S}}(f):=\sup _{Q \in \mathcal{S}}\left(\left\langle|f|^{p}\right\rangle_{Q}^{u}\right)^{\frac{1}{p}}
$$

Then by duality, it suffices to give a characterization for the following two weight norm inequality

$$
\left\|T_{\tau}(f u)\right\|_{L^{q}(v)} \leq C\|f\|_{L^{p}(u)}
$$

where

$$
T_{\tau}(f)=\sum_{Q \in \mathcal{S}} \tau_{Q}\langle f\rangle_{Q} \mathbf{1}_{Q}
$$

and recall that

$$
\tau_{Q}=\langle u\rangle_{Q}^{-\frac{1}{p_{0}^{\prime}}}\langle v\rangle_{Q}^{-\frac{1}{q_{0}}} \frac{\lambda_{Q}}{|Q|}
$$

The case of $p \leq q$ was due to Lacey, Sawyer and Uriarte-Tuero [15], and the case of $p>q$ was given by Tanaka [21]. Here we follow the unified testing for both cases given by Hänninen, Hytönen and the author [8].

Proposition 2.4. [8, Theorem 1.5] Let $p, q \in(1, \infty)$ and $w, \sigma$ be two measures. Let

$$
\begin{aligned}
T(f \sigma) & :=\sum_{Q \in \mathcal{D}} \lambda_{Q} \int_{Q} f d \sigma \cdot 1_{Q}, \quad \lambda_{Q} \geq 0, \\
T_{Q}(f \sigma) & :=\sum_{\substack{Q^{\prime} \in \mathcal{D} \\
Q^{\prime} \subseteq Q}} \lambda_{Q^{\prime}} \int_{Q^{\prime}} f d \sigma \cdot 1_{Q^{\prime}} .
\end{aligned}
$$

We have

$$
\|T(\cdot \sigma)\|_{L^{p}(\sigma) \rightarrow L^{q}(w)} \approx \mathfrak{T}_{s}+\mathfrak{T}_{s}^{*}
$$

where $s \in(1, \infty]$ is determined by

$$
\frac{1}{s}=\left(\frac{1}{q}-\frac{1}{p}\right)_{+}:=\max \left\{\frac{1}{q}-\frac{1}{p}, 0\right\} .
$$

and

$$
\begin{aligned}
& \mathfrak{T}_{s}:=\sup _{\mathcal{F}}\left\|\left\{\frac{\left\|T_{F}(\sigma)\right\|_{L^{q}(w)}}{\sigma(F)^{1 / p}}\right\}_{F \in \mathcal{F}}\right\|_{\ell^{s}}, \\
& \mathfrak{T}_{s}^{*}:=\sup _{\mathcal{G}}\left\|\left\{\frac{\left\|T_{G}(w)\right\|_{L^{p^{\prime}}(\sigma)}}{w(G)^{1 / q^{\prime}}}\right\}_{G \in \mathcal{G}}\right\|_{\ell^{s}},
\end{aligned}
$$

where the supremums are taken over all subcollections $\mathcal{F}$ and $\mathcal{G}$ of $\mathcal{S}$ that are sparse with respect to $\sigma$ and $\omega$, respectively. 
Now combining (2.3) and Proposition 2.4, Theorem 1.2 follows immediately.

\section{MiXed $A_{p}-A_{\infty}$ TyPe estimate for Bilinear forms}

In this section, we focus on the sharp constant for the case $p=q$. In this case, the testing condition degenerate to the Sawyer type testing condition. Namely,

$$
\mathcal{N} \approx \sup _{R \in \mathcal{S}} \frac{\left\|T_{R}(v)\right\|_{L^{p^{\prime}}(u)}}{v(R)^{1 / p^{\prime}}}+\sup _{R \in \mathcal{S}} \frac{\left\|T_{R}(u)\right\|_{L^{p}(v)}}{u(R)^{1 / p}} .
$$

Before further estimates, we introduce the following proposition.

Proposition 3.2. Let $\mathcal{S}$ be a sparse family and $0 \leq \gamma, \eta<1$ satisfying $\gamma+\eta<1$. Then

$$
\sum_{\substack{Q \in \mathcal{S} \\ Q \subset R}}\langle u\rangle_{Q}^{\gamma}\langle v\rangle_{Q}^{\eta}|Q| \lesssim\langle u\rangle_{R}^{\gamma}\langle v\rangle_{R}^{\eta}|R| .
$$

Proof. Indeed, set $1 / r:=\gamma+\eta, 1 / s:=\gamma+(1-1 / r) / 2$ and $1 / s^{\prime}:=1-1 / s$. Denote

$$
E_{Q}:=Q \backslash \bigcup_{Q^{\prime} \in \mathcal{S}, Q^{\prime} \subsetneq Q} Q^{\prime} .
$$

By sparseness and Kolmogorov's inequality, we have

$$
\begin{aligned}
\sum_{\substack{Q \in \mathcal{S} \\
Q \subset R}}\langle u\rangle_{Q}^{\gamma}\langle v\rangle_{Q}^{\eta}|Q| & \leq 2 \sum_{\substack{Q \in \mathcal{S} \\
Q \subset R}}\langle u\rangle_{Q}^{\gamma}\langle v\rangle_{Q}^{\eta}\left|E_{Q}\right| \\
& \leq 2 \int_{R} M\left(u \mathbf{1}_{R}\right)^{\gamma} M\left(v \mathbf{1}_{R}\right)^{\eta} d x \\
& \leq 2\left(\int_{R} M\left(u \mathbf{1}_{R}\right)^{s \gamma}\right)^{1 / s}\left(\int_{R} M\left(v \mathbf{1}_{R}\right)^{s^{\prime} \eta}\right)^{1 / s^{\prime}} \\
& \lesssim\langle u\rangle_{R}^{\gamma}|R|^{1 / s}\langle v\rangle_{R}^{\eta}|R|^{1 / s^{\prime}} \\
& =\langle u\rangle_{R}^{\gamma}\langle v\rangle_{R}^{\eta}|R|
\end{aligned}
$$

We also need the following result

Proposition 3.3 ([3], Proposition 2.2). Let $1<s<\infty, \sigma$ be a positive Borel measure and

$$
\phi=\sum_{Q \in \mathcal{D}} \alpha_{Q} \mathbf{1}_{Q}, \quad \phi_{Q}=\sum_{Q^{\prime} \subset Q} \alpha_{Q^{\prime}} \mathbf{1}_{Q^{\prime}} .
$$

Then

$$
\|\phi\|_{L^{s}(\sigma)} \approx\left(\sum_{Q \in \mathcal{D}} \alpha_{Q}\left(\left\langle\phi_{Q}\right\rangle_{Q}^{\sigma}\right)^{s-1} \sigma(Q)\right)^{1 / s} .
$$

Now we are ready to prove Theorem 1.6. 
Proof of Theorem 1.6. As that in [2], we denote by $\rho$ the critical index $1+$ $p_{0} / q_{0}^{\prime}$. First, we consider the case $p \geq \rho$. set

$$
\alpha=\frac{1}{p_{0}(r-1)} \min \left\{p-p_{0}, 1\right\} .
$$

We can check that

$$
\frac{1}{p_{0}}-(r-1) \alpha \geq 0, \frac{1}{q_{0}^{\prime}}-\alpha \geq 0, \text { and } \frac{1}{p_{0}}-(r-1) \alpha+\frac{1}{q_{0}^{\prime}}-\alpha<1 .
$$

By Propositions 3.3 and 3.2, we have

$$
\begin{aligned}
& \left\|T_{R}(v)\right\|_{L^{p^{\prime}}(u)} \\
& =\left\|\sum_{\substack{Q \in \mathcal{S} \\
Q \subset R}}\langle u\rangle_{Q}^{-\frac{1}{p_{0}^{\prime}}}\langle v\rangle_{Q}^{\frac{1}{q_{0}^{\prime}}} \mathbf{1}_{Q}\right\|_{L^{p^{\prime}}(u)} \\
& \bar{\sim}\left(\sum_{\substack{Q \in \mathcal{S} \\
Q \subset R}}\langle u\rangle_{Q}^{-\frac{1}{p_{0}^{\prime}}}\langle v\rangle_{Q}^{\frac{1}{q_{0}^{\prime}}} u(Q)\left(\frac{1}{u(Q)} \sum_{Q^{\prime} \subset Q}\langle u\rangle_{Q^{\prime}}^{-\frac{1}{p_{0}^{\prime}}}\langle v\rangle_{Q_{0}^{\prime}}^{\frac{1}{q_{0}^{\prime}}} u\left(Q^{\prime}\right)\right)^{p^{\prime}-1}\right)^{\frac{1}{p^{\prime}}} \\
& \leq[v, u]_{A_{r}}^{\frac{\alpha}{p}}\left(\sum_{\substack{Q \in \mathcal{S} \\
Q \subset R}}\langle u\rangle_{Q}^{-\frac{1}{p_{0}^{\prime}}}\langle v\rangle_{Q}^{\frac{1}{q_{0}^{\prime}}} u(Q)\left(\frac{1}{u(Q)} \sum_{Q^{\prime} \subset Q}\langle u\rangle_{Q^{\prime}}^{\frac{1}{p_{0}}-(r-1) \alpha}\langle v\rangle_{Q^{\prime}}^{\frac{1}{q_{0}^{\prime}}-\alpha}\left|Q^{\prime}\right|\right)^{p^{\prime}-1}\right)^{\frac{1}{p^{\prime}}} \\
& \lesssim[v, u]_{A_{r}}^{\frac{\alpha}{p}}\left(\sum_{\substack{Q \in \mathcal{S} \\
Q \subset R}}\langle u\rangle_{Q}^{-\frac{1}{p_{0}^{\prime}}}\langle v\rangle_{Q}^{\frac{1}{q_{0}^{\prime}}} u(Q)\left(\frac{1}{u(Q)}\langle u\rangle_{Q}^{\frac{1}{p_{0}}-(r-1) \alpha}\langle v\rangle_{Q}^{\frac{1}{q_{0}^{\prime}}-\alpha}|Q|\right)^{p^{\prime}-1}\right)^{\frac{1}{p^{\prime}}} \\
& =[v, u]_{A_{r}}^{\frac{\alpha}{p}}\left(\sum_{\substack{Q \in \mathcal{S} \\
Q \subset R}}\langle u\rangle_{Q}^{\frac{1}{p_{0}}-\left(p^{\prime}-1\right)\left((r-1) \alpha+\frac{1}{p_{0}^{\prime}}\right)}\langle v\rangle_{Q}^{\frac{1}{q_{0}^{\prime}}+\left(\frac{1}{q_{0}^{\prime}}-\alpha\right)\left(p^{\prime}-1\right)}|Q|\right)^{\frac{1}{p^{\prime}}} \\
& \leq[v, u]_{A_{r}}^{\frac{\alpha}{p}+\frac{1}{p^{\prime} p_{0}(r-1)}-\left(\frac{\alpha}{p}+\frac{1}{p p_{0}^{\prime}(r-1)}\right)}\left(\sum_{\substack{Q \in \mathcal{S} \\
Q \subset R}} v(Q)\right)^{\frac{1}{p^{\prime}}} \\
& \lesssim[v, u]_{A_{r}}^{\frac{1}{q_{0}^{\prime}}-\frac{1}{p^{\prime}}}[v]_{A_{\infty}}^{\frac{1}{p^{\prime}}} v(R)^{\frac{1}{p^{\prime}}},
\end{aligned}
$$

For the case $p<\rho$, set

$$
\tilde{\alpha}=\left(\frac{1}{q_{0}^{\prime}}-\frac{1}{p^{\prime}}\right) p
$$

Again, we can check that

$$
\begin{gathered}
\frac{1}{q_{0}^{\prime}}-\tilde{\alpha}=\frac{1}{q_{0}^{\prime}}\left(p^{\prime}-1\right)(p-1)-\frac{1}{q_{0}^{\prime}}\left(p^{\prime}-q_{0}^{\prime}\right)(p-1) \geq 0, \\
\frac{1}{p_{0}}-\tilde{\alpha}(r-1) \geq 0,
\end{gathered}
$$


and

$$
\frac{1}{p_{0}}-\tilde{\alpha}(r-1)+\frac{1}{q_{0}^{\prime}}-\tilde{\alpha}=1-(p-1)\left(\frac{1}{p_{0}}-\frac{1}{q_{0}}\right)<1 .
$$

By Propositions 3.3 and 3.2 again, we have

$$
\begin{aligned}
& \left\|T_{R}(v)\right\|_{L^{p^{\prime}}(u)} \\
& \bar{\sim}\left(\sum_{\substack{Q \in \mathcal{S} \\
Q \subset R}}\langle u\rangle_{Q}^{-\frac{1}{p_{0}^{\prime}}}\langle v\rangle_{Q}^{\frac{1}{q_{0}^{\prime}}} u(Q)\left(\frac{1}{u(Q)} \sum_{Q^{\prime} \subset Q}\langle u\rangle_{Q^{\prime}}^{\frac{1}{p_{0}}}\langle v\rangle_{Q^{\prime}}^{\frac{1}{q_{0}^{\prime}}}\left|Q^{\prime}\right|\right)^{p^{\prime}-1}\right)^{\frac{1}{p^{\prime}}} \\
& \leq[v, u]_{A_{r}}^{\frac{\tilde{\alpha}}{p}}\left(\sum_{\substack{Q \in \mathcal{S} \\
Q \subset R}}\langle u\rangle_{Q}^{-\frac{1}{p_{0}^{\prime}}}\langle v\rangle_{Q}^{\frac{1}{q_{0}^{\prime}}} u(Q)\left(\frac{1}{u(Q)} \sum_{Q^{\prime} \subset Q}\langle u\rangle_{Q^{\prime}}^{\frac{1}{p_{0}}-\tilde{\alpha}(r-1)}\langle v\rangle_{Q^{\prime}}^{\frac{1}{q_{0}^{\prime}}-\tilde{\alpha}}\left|Q^{\prime}\right|\right)^{p^{\prime}-1}\right)^{\frac{1}{p^{\prime}}} \\
& \lesssim[v, u]_{A_{r}}^{\frac{\tilde{\alpha}}{p}}\left(\sum_{\substack{Q \in \mathcal{S} \\
Q \subset R}}\langle u\rangle_{Q}^{-\frac{1}{p_{0}^{\prime}}}\langle v\rangle_{Q}^{\frac{1}{q_{0}^{\prime}}} u(Q)\left(\frac{1}{u(Q)}\langle u\rangle_{Q}^{\frac{1}{p_{0}}-\tilde{\alpha}(r-1)}\langle v\rangle_{Q}^{\frac{1}{q_{0}^{\prime}}-\tilde{\alpha}}|Q|\right)^{p^{\prime}-1}\right)^{\frac{1}{p^{\prime}}} \\
& =[v, u]_{A_{r}}^{\frac{\tilde{\alpha}}{p}}\left(\sum_{\substack{Q \in \mathcal{S} \\
Q \subset R}}\langle u\rangle_{Q}^{\frac{1}{p_{0}}-\left(p^{\prime}-1\right)\left(\frac{1}{p_{0}^{\prime}}+\tilde{\alpha}(r-1)\right)}\langle v\rangle_{Q}^{\frac{1}{q_{0}^{\prime}}+\left(p^{\prime}-1\right)\left(\frac{1}{q_{0}^{\prime}}-\tilde{\alpha}\right)}|Q|\right)^{\frac{1}{p^{\prime}}} \\
& =[v, u]_{A_{r}}^{\frac{1}{q_{0}^{\prime}}-\frac{1}{p^{\prime}}}\left(\sum_{\substack{Q \in \mathcal{S} \\
Q \subset R}} v(Q)\right)^{\frac{1}{p^{\prime}}} \\
& \lesssim[v, u]_{A_{r}}^{\frac{1}{q^{\prime}}-\frac{1}{p^{\prime}}}[v]_{A_{\infty}}^{\frac{1}{p^{\prime}}} v(R)^{\frac{1}{p^{\prime}}} .
\end{aligned}
$$

By symmetry, we have

$$
\begin{aligned}
\left\|T_{R}(u)\right\|_{L^{p}(v)} & \lesssim[u, v]_{A_{r^{\prime}}}^{\frac{1}{p_{0}}-\frac{1}{p}}[u]_{A_{\infty}}^{\frac{1}{p}} u(R)^{\frac{1}{p}} \\
& =[v, u]_{A_{r}}^{\frac{1}{q_{0}^{\prime}}-\frac{1}{p^{\prime}}}[u]_{A_{\infty}}^{\frac{1}{p}} u(R)^{\frac{1}{p}} .
\end{aligned}
$$

Then the desired estimate follows from (3.1) immediately.

\section{Mixed $A_{p}-A_{\infty}$ Type estimates With one supremum}

In this section, we study the one supremum estimate. And this could be done by just slightly modify the arguments in the previous section. We first prove Theorem 1.8.

Proof of Theorem 1.8. By symmetry, we only need to estimate $\left\|T_{R}(v)\right\|_{L^{p^{\prime}}(u)}$. Denote

$$
\mathcal{S}_{a}=\left\{Q \in \mathcal{S}: 2^{a} \leq A_{\infty}(v, Q)<2^{a+1}\right\} .
$$

We will abuse of using the notations $T$ and $T_{R}(v)$ slightly, which is now understood as summation over $\mathcal{S}_{a}$ instead of $\mathcal{S}$. We still consider the case 
$p \geq \rho$ first, we have

$$
\begin{aligned}
& \left\|T_{R}(v)\right\|_{L^{p^{\prime}}(u)} \\
& \bar{\sim}\left(\sum_{\substack{Q \in \mathcal{S}_{a} \\
Q \subset R}}\langle u\rangle_{Q}^{-\frac{1}{p_{0}^{\prime}}}\langle v\rangle_{Q}^{\frac{1}{q_{0}^{\prime}}} u(Q)\left(\frac{1}{u(Q)} \sum_{Q^{\prime} \subset Q}\langle u\rangle_{Q^{\prime}}^{\frac{1}{p_{0}}}\langle v\rangle_{Q^{\prime}}^{\frac{1}{q^{\prime}}}\left|Q^{\prime}\right|\right)^{p^{\prime}-1}\right)^{\frac{1}{p^{\prime}}} \\
& \leq\left(\frac{[v, u]_{\left(A_{r}\right)\left(\phi\left(A_{\infty}\right)\right)}}{2^{\frac{a}{p^{\prime}}} \phi\left(2^{a}\right)}\right)^{\frac{\alpha q_{0}}{q_{0}-p}}\left(\sum _ { \substack { Q \in \mathcal { S } _ { a } \\
Q \subset R } } \langle u \rangle _ { Q } ^ { - \frac { 1 } { p _ { 0 } ^ { \prime } } } \langle v \rangle _ { Q } ^ { \frac { 1 } { q _ { 0 } ^ { \prime } } } u ( Q ) \left(\frac{1}{u(Q)}\right.\right. \\
& \left.\left.\times \sum_{Q^{\prime} \subset Q}\langle u\rangle_{Q^{\prime}}^{\frac{1}{p_{0}}-(r-1) \alpha}\langle v\rangle_{Q_{0}^{\prime}}^{\frac{1}{q^{\prime}}-\alpha}\left|Q^{\prime}\right|\right)^{p^{\prime}-1}\right)^{\frac{1}{p^{\prime}}} \\
& \lesssim\left(\frac{[v, u]_{\left(A_{r}\right)\left(\phi\left(A_{\infty}\right)\right)}}{2^{\frac{a}{p^{\prime}}} \phi\left(2^{a}\right)}\right)^{\frac{\alpha q_{0}}{q_{0}-p}}\left(\sum_{\substack{Q \in \mathcal{S}_{a} \\
Q \subset R}}\langle u\rangle_{Q}^{-\frac{1}{p_{0}^{\prime}}}\langle v\rangle_{Q}^{\frac{1}{q_{0}^{\prime}}} u(Q)\right. \\
& \left.\times\left(\frac{1}{u(Q)}\langle u\rangle_{Q}^{\frac{1}{p_{0}}-(r-1) \alpha}\langle v\rangle_{Q}^{\frac{1}{q_{0}^{\prime}}-\alpha}|Q|\right)^{p^{\prime}-1}\right)^{\frac{1}{p^{\prime}}} \\
& =\left(\frac{[v, u]_{\left(A_{r}\right)\left(\phi\left(A_{\infty}\right)\right)}}{2^{\frac{a}{p^{\prime}}} \phi\left(2^{a}\right)}\right)^{\frac{\alpha q_{0}}{q_{0}-p}}\left(\sum_{\substack{Q \in \mathcal{S}_{a} \\
Q \subset R}}\langle u\rangle_{Q}^{\frac{1}{p_{0}}-\left(p^{\prime}-1\right)\left((r-1) \alpha+\frac{1}{p_{0}^{\prime}}\right)}\langle v\rangle_{Q}^{\frac{1}{q_{0}^{\prime}}+\left(\frac{1}{q_{0}^{\prime}}-\alpha\right)\left(p^{\prime}-1\right)}|Q|\right)^{\frac{1}{p^{\prime}}} \\
& \leq \frac{[v, u]_{\left(A_{r}\right)\left(\phi\left(A_{\infty}\right)\right)}}{2^{\frac{a}{p^{\prime}}} \phi\left(2^{a}\right)}\left(\sum_{\substack{Q \in \mathcal{S}_{a} \\
Q \subset R}} v(Q)\right)^{\frac{1}{p^{\prime}}} \\
& \lesssim \frac{1}{\phi\left(2^{a}\right)}[v, u]_{\left(A_{r}\right)\left(\phi\left(A_{\infty}\right)\right)} v(R)^{\frac{1}{p^{\prime}}} .
\end{aligned}
$$

For the case $p<\rho$, similar arguments show that

$$
\left\|T_{R}(v)\right\|_{L^{p^{\prime}}(u)} \lesssim \frac{1}{\phi\left(2^{a}\right)}[v, u]_{\left(A_{r}\right)\left(\phi\left(A_{\infty}\right)\right)} v(R)^{\frac{1}{p^{\prime}}} .
$$

It remains to sum over $a$, by definition, $a \geq 0$, we have

$$
\begin{aligned}
\sup _{R \in \mathcal{S}} \frac{\left\|T_{R}(v)\right\|_{L^{p^{\prime}}(u)}}{v(R)^{1 / p^{\prime}}} & \lesssim \sum_{a \geq 0} \frac{1}{\phi\left(2^{a}\right)}[v, u]_{\left(A_{r}\right)\left(\phi\left(A_{\infty}\right)\right)} \\
& \lesssim \sum_{a \geq 0} \int_{2^{a-1}}^{2^{a}} \frac{1}{\phi(t)} \frac{d t}{t}[v, u]_{\left(A_{r}\right)\left(\phi\left(A_{\infty}\right)\right)} \\
& =\int_{1 / 2}^{\infty} \frac{1}{\phi(t)} \frac{d t}{t}[v, u]_{\left(A_{r}\right)\left(\phi\left(A_{\infty}\right)\right) .}
\end{aligned}
$$

This completes the proof.

Next we prove Theorem 1.9. 
Proof of Theorem 1.9. We follow the same strategy as that in the proof of Theorem 1.8. Set

$$
\mathcal{S}_{a}=\left\{Q \in \mathcal{S}: 2^{a} \leq A_{\infty}^{\exp }(v, Q)<2^{a+1}\right\} .
$$

We have

$$
\begin{aligned}
\left\|T_{R}(v)\right\|_{L^{p^{\prime}}(u)} & \lesssim \sum_{a \geq 0} \frac{[v, u]_{\left(A_{r}\right)\left(\Phi\left(A_{\infty}\right)\right)}}{2^{\frac{a}{p^{\prime}}} \Phi\left(2^{a}\right)}\left(\sum_{\substack{Q \in \mathcal{S}_{a} \\
Q \subset R}} v(Q)\right)^{\frac{1}{p^{\prime}}} \\
& \leq[v, u]_{\left(A_{r}\right)\left(\Phi\left(A_{\infty}\right)\right)} \sum_{a \geq 0} \frac{1}{\Phi\left(2^{a}\right)}\left(\sum_{\substack{Q \in \mathcal{S}_{a} \\
Q \subset R}} \exp \left(f_{Q} \log v\right)|Q|\right)^{\frac{1}{p^{\prime}}} \\
& \leq[v, u]_{\left(A_{r}\right)\left(\Phi\left(A_{\infty}\right)\right)}\left(\sum_{a \geq 0} \frac{1}{\Phi\left(2^{a}\right)^{p}}\right)^{\frac{1}{p}}\left(\sum_{\substack{Q \in \mathcal{S} \\
Q \subset R}} \exp \left(f_{Q} \log v\right)|Q|\right)^{\frac{1}{p^{\prime}}} \\
& \lesssim[v, u]_{\left(A_{r}\right)\left(\Phi\left(A_{\infty}\right)\right)} v(R)^{\frac{1}{p^{\prime}}}\left(\int_{1 / 2}^{\infty} \frac{1}{\Phi(t)^{p}} \frac{d t}{t}\right)^{\frac{1}{p}} .
\end{aligned}
$$

This completes the proof.

\section{FURTHER DISCUSSIONS}

In this section, we propose a new conjecture which implies both the one supremum conjecture (with $A_{\infty}$ replaced by $A_{\infty}^{\exp }$ ) and the separated bump conjecture. On the other hand, it shares many similar results as the later two problems. To be precise, define

$$
\begin{gathered}
{[u, v]_{A, q_{0}, p, r}=\sup _{Q}\langle v\rangle_{Q}^{\frac{1}{p}-\frac{1}{q_{0}}}\langle u\rangle_{Q}^{\left(\frac{1}{p}-\frac{1}{q_{0}}\right)(r-1)} \frac{\langle u\rangle_{Q}^{\frac{1}{p}}}{\left\langle u^{\frac{1}{p}}\right\rangle_{A, Q}},} \\
{[v, u]_{B, p_{0}^{\prime}, p^{\prime}, r^{\prime}}=\sup _{Q}\langle u\rangle_{Q}^{\frac{1}{p^{\prime}}-\frac{1}{p_{0}^{\prime}}}\langle v\rangle_{Q}^{\left(\frac{1}{p^{\prime}}-\frac{1}{p_{0}^{\prime}}\right)\left(r^{\prime}-1\right)} \frac{\langle v\rangle_{Q}^{\frac{1}{p^{\prime}}}}{\left\langle v^{\frac{1}{p^{\prime}}}\right\rangle_{B, Q}},}
\end{gathered}
$$

where $A \in B_{p}$ and $B \in B_{p^{\prime}}$. Recall that we say a Young function $A$ belongs to $B_{p}$ if

$$
\int_{1 / 2}^{\infty} \frac{A(t)}{t^{p}} \frac{d t}{t}<\infty
$$

and the Luxembourg norm $\langle f\rangle_{A, Q}$ is defined by

$$
\langle f\rangle_{A, Q}:=\inf \left\{\lambda>0:\langle A(f / \lambda)\rangle_{Q} \leq 1\right\} .
$$

We propose the following conjecture.

Conjecture 5.1. Let $(w, \sigma)$ be a pair of weights, $\lambda_{Q}=|Q|, p=q$ and $\mathcal{N}$ be the best constant such that (1.3) holds. Let $A \in B_{p}$ and $B \in B_{p^{\prime}}$. Then there holds

$$
\mathcal{N} \leq C\left([u, v]_{A, q_{0}, p, r}+[v, u]_{B, p_{0}^{\prime}, p^{\prime}, r^{\prime}}\right)
$$


where the constant $C>0$ is independent of $w$ and $\sigma$.

Now we shall see that this conjecture implies both the one supremum conjecture and also the separated bump conjecture. For general indices $p_{0}$ and $q_{0}$, the separated bump conjecture can be stated as follows

Conjecture 5.2. Let $(w, \sigma)$ be a pair of weights, $\lambda_{Q}=|Q|, p=q$ and $\mathcal{N}$ be the best constant such that (1.3) holds. Let $A \in B_{p}$ and $B \in B_{p^{\prime}}$. Then there holds

$$
\mathcal{N} \leq C\left((u, v)_{A, q_{0}, p, r}+(v, u)_{B, p_{0}^{\prime}, p^{\prime}, r^{\prime}}\right),
$$

where the constant $C>0$ is independent of $w$ and $\sigma$ and

$$
\begin{gathered}
(u, v)_{A, q_{0}, p, r}=\sup _{Q}\langle v\rangle_{Q}^{\frac{1}{p}-\frac{1}{q_{0}}}\langle u\rangle_{Q}^{\left(\frac{1}{p}-\frac{1}{q_{0}}\right)(r-1)} \frac{\left\langle u^{\frac{1}{p^{\prime}}}\right\rangle_{\bar{A}, Q}}{\langle u\rangle_{Q}^{\frac{1}{p^{\prime}}}}, \\
(v, u)_{B, p_{0}^{\prime}, p^{\prime}, r^{\prime}}=\sup _{Q}\langle u\rangle_{Q}^{\frac{1}{p^{\prime}}-\frac{1}{p_{0}^{\prime}}}\langle v\rangle_{Q}^{\left(\frac{1}{p^{\prime}}-\frac{1}{p_{0}^{\prime}}\right)\left(r^{\prime}-1\right)} \frac{\left\langle v^{\frac{1}{p}}\right\rangle_{\bar{B}, Q}}{\langle v\rangle_{Q}^{\frac{1}{p}}} .
\end{gathered}
$$

Indeed, by general Hölder's inequality,

$$
\frac{\langle u\rangle_{Q}^{\frac{1}{p}}}{\left\langle u^{\frac{1}{p}}\right\rangle_{A, Q}} \leq \frac{\left\langle u^{\frac{1}{p^{\prime}}}\right\rangle_{\bar{A}, Q}}{\langle u\rangle_{Q}^{\frac{1}{p^{\prime}}}}, \frac{\langle v\rangle_{Q}^{\frac{1}{p^{\prime}}}}{\left\langle v^{\frac{1}{p^{\prime}}}\right\rangle_{B, Q}} \leq \frac{\left\langle v^{\frac{1}{p}}\right\rangle_{\bar{B}, Q}}{\langle v\rangle_{Q}^{\frac{1}{p}}}
$$

which means Conjecture 5.1 implies separated bump conjecture. On the other hand, by Jensen's inequality,

$$
\left\langle u^{\frac{1}{p}}\right\rangle_{A, Q} \geq \exp \left(f_{Q} \log u^{\frac{1}{p}}\right)=\left(\exp \left(f_{Q} \log u\right)\right)^{\frac{1}{p}} .
$$

Then

$$
\frac{\langle u\rangle_{Q}^{\frac{1}{p}}}{\left\langle u^{\frac{1}{p}}\right\rangle_{A, Q}} \leq A_{\infty}^{\exp }(u, Q)^{\frac{1}{p}},
$$

which means Conjecture 5.1 implies also the one supremum conjecture.

Although Conjecture 5.1 is stronger than both separated bump conjecture and one supremum conjecture, it still contain the essential property as the separated bump conjecture and one supremum conjecture. Indeed, we are free to write

$$
\left\langle\sigma^{\frac{1}{p}}\right\rangle_{A, Q}=\langle\sigma\rangle_{\tilde{A}, Q}^{\frac{1}{p}}
$$

where $\tilde{A}(t)=A\left(t^{1 / p}\right)$. Since $M_{A}^{\mathcal{D}}$ is bounded on $L^{p}$ (see [20]), we have $M_{\tilde{A}}^{\mathcal{D}}$ is bounded on $L^{1}$, and this is the key point. We shall see this by showing the following result. 
Theorem 5.3. Let $(w, \sigma)$ be a pair of weights, $\lambda_{Q}=|Q|, p=q$ and $\mathcal{N}$ be the best constant such that (1.3) holds. Let $A \in B_{p}, B \in B_{p^{\prime}}$ and $\phi, \psi$ be increasing functions such that

$$
\int_{1 / 2}^{\infty}\left(\frac{1}{\phi(t)^{p}}+\frac{1}{\psi(t)^{p^{\prime}}}\right) \frac{d t}{t}<\infty
$$

Then

where

$$
\mathcal{N} \lesssim[u, v]_{A, q_{0}, p, r, \psi}+[v, u]_{B, p_{0}^{\prime}, p^{\prime}, r^{\prime}, \phi}
$$

$$
\begin{gathered}
{[u, v]_{A, q_{0}, p, r, \psi}=\sup _{Q}\langle v\rangle_{Q}^{\frac{1}{p}-\frac{1}{q_{0}}}\langle u\rangle_{Q}^{\left(\frac{1}{p}-\frac{1}{q_{0}}\right)(r-1)} \frac{\langle u\rangle_{Q}^{\frac{1}{p}}}{\left\langle u^{\frac{1}{p}}\right\rangle_{A, Q}} \psi\left(\frac{\langle u\rangle_{Q}^{\frac{1}{p}}}{\left\langle u^{\frac{1}{p}}\right\rangle_{A, Q}}\right),} \\
{[v, u]_{B, p_{0}^{\prime}, p^{\prime}, r^{\prime}, \phi}=\sup _{Q}\langle u\rangle_{Q}^{\frac{1}{p^{\prime}}-\frac{1}{p_{0}^{\prime}}}\langle v\rangle_{Q}^{\left(\frac{1}{p^{\prime}}-\frac{1}{p_{0}^{\prime}}\right)\left(r^{\prime}-1\right)} \frac{\langle v\rangle_{Q}^{\frac{1}{p^{\prime}}}}{\left\langle v^{\left.\frac{1}{p^{\prime}}\right\rangle_{B, Q}} \phi\left(\frac{\langle v\rangle_{Q}^{\frac{1}{p^{\prime}}}}{\left\langle v^{\frac{1}{p^{p}}}\right\rangle_{B, Q}}\right) .\right.} .}
\end{gathered}
$$

Proof. The proof is quite similar to the proof of Theorem 1.9. Set

$$
\mathcal{S}_{a}:=\left\{Q \in \mathcal{S}: 2^{a} \leq \frac{\langle v\rangle_{Q}^{\frac{1}{p^{\prime}}}}{\left\langle v^{\frac{1}{p^{\prime}}}\right\rangle_{B, Q}}<2^{a+1}\right\} .
$$

Then we have

$$
\begin{aligned}
& \left\|T_{R}(v)\right\|_{L^{p^{\prime}}(u)} \lesssim \sum_{a \geq 0} \frac{[v, u]_{B, p_{0}^{\prime}, p^{\prime}, r^{\prime}, \phi}}{2^{a} \phi\left(2^{a}\right)}\left(\sum_{\substack{Q \in \mathcal{S}_{a} \\
Q \subset R}} v(Q)\right)^{\frac{1}{p^{\prime}}} \\
& \leq[v, u]_{B, p_{0}^{\prime}, p^{\prime}, r^{\prime}, \phi} \sum_{a \geq 0} \frac{1}{\phi\left(2^{a}\right)}\left(\sum_{\substack{Q \in \mathcal{S}_{a} \\
Q \subset R}}\left\langle v^{\frac{1}{p^{\prime}}}\right\rangle_{B, Q}^{p^{\prime}}|Q|\right)^{\frac{1}{p^{\prime}}} \\
& \leq[v, u]_{B, p_{0}^{\prime}, p^{\prime}, r^{\prime}, \phi}\left(\sum_{a \geq 0} \frac{1}{\phi\left(2^{a}\right)^{p}}\right)^{\frac{1}{p}}\left(\sum_{\substack{Q \in \mathcal{S} \\
Q \subset R}}\left\langle v^{\frac{1}{p^{\prime}}}\right\rangle_{B, Q}^{p^{\prime}}|Q|\right)^{\frac{1}{p^{\prime}}} \\
& \lesssim[v, u]_{B, p_{0}^{\prime}, p^{\prime}, r^{\prime}, \phi} v(R)^{\frac{1}{p^{\prime}}}\left\|M_{B}\right\|_{L^{p^{\prime} \rightarrow L^{p^{\prime}}}}\left(\int_{1 / 2}^{\infty} \frac{1}{\phi(t)^{p}} \frac{d t}{t}\right)^{\frac{1}{p}} .
\end{aligned}
$$

Then the desired result follows immediately from Theorem 1.2.

Remark 5.4. In [14], Lacey showed the same result with $\langle u\rangle_{Q}^{\frac{1}{p}} /\left\langle u^{\frac{1}{p}}\right\rangle_{A, Q}$ replaced by $\left\langle u^{\frac{1}{p^{\prime}}}\right\rangle_{\bar{A}, Q} /\langle u\rangle_{Q}^{\frac{1}{p^{\prime}}}$ and analogously for $v$ when $p_{0}=1$ and $q_{0}=\infty$ (Recall that in this case, $u=\sigma$ and $v=w$ ). And therefore, our estimate improves Lacey's bound. On the other hand, it is easy to see that it improves Theorem 1.9 as well.

We also have the following result. 
Theorem 5.5. Let $(w, \sigma)$ be a pair of weights, $\lambda_{Q}=|Q|, p=q$ and $\mathcal{N}$ be the best constant such that (1.3) holds. Let $A \in B_{p}, B \in B_{p^{\prime}}$. Then

$$
\mathcal{N} \lesssim[u, v]_{A, B}
$$

where

$$
[u, v]_{A, B}=\sup _{Q}\langle v\rangle_{Q}^{\frac{1}{p}-\frac{1}{q_{0}}}\langle u\rangle_{Q}^{\left(\frac{1}{p}-\frac{1}{q_{0}}\right)(r-1)} \frac{\langle u\rangle_{Q}^{\frac{1}{p}}}{\left\langle u^{\frac{1}{p}}\right\rangle_{A, Q}} \cdot \frac{\langle v\rangle_{Q}^{\frac{1}{p^{\prime}}}}{\left\langle v^{\frac{1}{p^{\prime}}}\right\rangle_{B, Q}} .
$$

Proof. Instead of using Proposition 3.3, we shall use the technique of parallel stopping cubes here. We define the principal cubes $\mathcal{F}$ for $(f, u)$ as follows

$$
\begin{aligned}
\mathcal{F} & :=\bigcup_{k=0}^{\infty} \mathcal{F}_{k}, \quad \mathcal{F}_{0}:=\{\text { maximal cubes in } \mathcal{S}\} \\
\mathcal{F}_{k+1} & \left.:=\bigcup_{F \in \mathcal{F}_{k}} \operatorname{ch}_{\mathcal{F}}(\mathrm{F}), \quad \operatorname{ch}_{\mathcal{F}}(\mathrm{F}):=\left\{\mathrm{Q} \subsetneq \mathrm{F} \text { maximal s.t. }\langle\mathrm{f}\rangle_{\mathrm{Q}}^{\mathrm{u}}>2\langle\mathrm{f}\rangle\right\rangle_{\mathrm{F}}^{\mathrm{u}}\right\},
\end{aligned}
$$

and analogously define $\mathcal{G}$ for $(g, v)$. We also denote by $\pi_{\mathcal{F}}(Q)$ the minimal cube in $\mathcal{F}$ which contains $Q$, and $\pi(Q)=(F, G)$ if $\pi_{\mathcal{F}}(Q)=F$ and $\pi_{\mathcal{G}}(Q)=$ $G$. By construction, we have

$$
\sum_{F \in \mathcal{F}}\left(\langle f\rangle_{F}^{u}\right)^{p} u(F) \lesssim\|f\|_{L^{p}(u)}^{p} .
$$

Now we start our arguments from (2.2). We have

$$
\begin{aligned}
\sum_{Q \in \mathcal{S}}\langle f\rangle_{Q}^{u}\langle g\rangle_{Q}^{v}\langle u\rangle_{Q}^{\frac{1}{p_{0}}}\langle v\rangle_{Q}^{\frac{1}{q_{0}^{\prime}}}|Q| & =\sum_{F \in \mathcal{F}} \sum_{G \in \mathcal{G}} \sum_{\pi(Q)=(F, G)}\langle f\rangle_{Q}^{u}\langle g\rangle_{Q}^{v}\langle u\rangle_{Q}^{\frac{1}{p_{0}}}\langle v\rangle_{Q}^{\frac{1}{q_{0}^{\prime}}}|Q| \\
& \lesssim \sum_{F \in \mathcal{F}}\langle f\rangle_{F}^{u} \sum_{\substack{G \in \mathcal{G} \\
G \subset F}}\langle g\rangle_{G}^{v} \sum_{\pi(Q)=(F, G)}\langle u\rangle_{Q}^{\frac{1}{p_{0}}}\langle v\rangle_{Q}^{\frac{1}{q_{0}^{\prime}}}|Q| \\
& +\sum_{G \in \mathcal{G}}\langle g\rangle_{G}^{v} \sum_{\substack{F \in \mathcal{F} \\
F \subset G}}\langle f\rangle_{F}^{u} \sum_{\pi(Q)=(F, G)}\langle u\rangle_{Q}^{\frac{1}{p_{0}}}\langle v\rangle_{Q}^{\frac{1}{q_{0}^{\prime}}}|Q| \\
& :=I+I I .
\end{aligned}
$$

By symmetry, we only need to estimate $I$. We have

$$
\begin{aligned}
& \sum_{\pi(Q)=(F, G)}\langle u\rangle_{Q}^{\frac{1}{p_{0}}}\langle v\rangle_{Q}^{\frac{1}{q_{0}^{\prime}}}|Q| \\
= & \sum_{\pi(Q)=(F, G)}\langle u\rangle_{Q}^{\frac{1}{p_{0}}-\frac{1}{p}}\langle v\rangle_{Q}^{\frac{1}{q_{0}^{\prime}}-\frac{1}{p^{\prime}}} \frac{\langle u\rangle_{Q}^{\frac{1}{p}}}{\left\langle u^{\frac{1}{p}}\right\rangle_{A, Q}} \cdot \frac{\langle v\rangle_{Q}^{\frac{1}{p^{\prime}}}}{\left\langle v^{\frac{1}{p^{\prime}}}\right\rangle_{B, Q}}\left\langle u^{\frac{1}{p}}\right\rangle_{A, Q} \cdot\left\langle v^{\frac{1}{p^{\prime}}}\right\rangle_{B, Q}|Q| \\
\leq & {[u, v]_{A, B}\left(\sum_{\pi(Q)=(F, G)}\left\langle u^{\frac{1}{p}}\right\rangle_{A, Q}^{p}|Q|\right)^{\frac{1}{p}}\left(\sum _ { \pi ( Q ) = ( F , G ) } \left\langlev^{\left.\left.\frac{1}{p^{\prime}}\right\rangle_{B, Q}^{p^{\prime}}|Q|\right)^{\frac{1}{p^{\prime}}}}\right.\right.}
\end{aligned}
$$




$$
\lesssim[u, v]_{A, B}\left(\sum_{\pi(Q)=(F, G)}\left\langle u^{\frac{1}{p}}\right\rangle_{A, Q}^{p}|Q|\right)^{\frac{1}{p}} v(G)^{\frac{1}{p^{\prime}}} .
$$

Then by Hölder's inequality,

$$
\begin{aligned}
I & \leq[u, v]_{A, B} \sum_{F \in \mathcal{F}}\langle f\rangle_{F}^{u}\left(\sum_{\substack{G \in \mathcal{G} \\
G \subset F}} \sum_{\pi(Q)=(F, G)}\left\langle u^{\frac{1}{p}}\right\rangle_{A, Q}^{p}|Q|\right)^{\frac{1}{p}}\left(\sum_{\substack{G \in \mathcal{G} \\
\pi_{\mathcal{F}}(G)=F}}\left(\langle g\rangle_{G}^{v}\right)^{p^{\prime}} v(G)\right)^{\frac{1}{p^{\prime}}} \\
& \lesssim[u, v]_{A, B} \sum_{F \in \mathcal{F}}\langle f\rangle_{F}^{u} u(F)^{\frac{1}{p}}\left(\sum_{\substack{G \in \mathcal{G} \\
\pi_{\mathcal{F}}(G)=F}}\left(\langle g\rangle_{G}^{v}\right)^{p^{\prime}} v(G)\right)^{\frac{1}{p^{\prime}}} \\
& \leq[u, v]_{A, B}\left(\sum_{F \in \mathcal{F}}\left(\langle f\rangle_{F}^{u}\right)^{p} u(F)\right)^{\frac{1}{p}}\left(\sum_{F \in \mathcal{F}} \sum_{\substack{G \in \mathcal{G} \\
\pi_{\mathcal{F}}(G)=F}}\left(\langle g\rangle_{G}^{v}\right)^{p^{\prime}} v(G)\right)^{\frac{1}{p^{\prime}}} \\
& \lesssim[u, v]_{A, B}\|f\|_{L^{p}(u)}\|g\|_{L^{p^{\prime}}(v)},
\end{aligned}
$$

where (5.6) is used in the last step.

Remark 5.7. In [18], Lerner proved the so-called bump conjecture, i.e., the same result with $\langle u\rangle_{Q}^{\frac{1}{p}} /\left\langle u^{\frac{1}{p}}\right\rangle_{A, Q}$ replaced by $\left\langle u^{\frac{1}{p^{\prime}}}\right\rangle_{\bar{A}, Q} /\langle u\rangle_{Q}^{\frac{1}{p^{\prime}}}$ and analogously for $v$ when $p_{0}=1$ and $q_{0}=\infty$. Therefore, our result improves the bump theorem. In [19], Lerner and Moen also proved the following estimate, for any Calderón-Zygmund operator $T$, there holds

$$
\begin{aligned}
\|T\|_{L^{p}(w)} & \leq \sup _{Q}\left(\langle w\rangle_{Q}\left\langle w^{1-p^{\prime}}\right\rangle_{Q}^{p-1}\right)^{\frac{1}{p-1}} A_{\infty}^{\exp }(w)(Q)^{1-\frac{1}{p-1}} \\
& =\sup _{Q}\langle w\rangle_{Q}^{\frac{1}{p}}\left\langle w^{1-p^{\prime}}\right\rangle_{Q}^{\frac{1}{p^{\prime}}} A_{\infty}^{\exp }(w, Q)^{\frac{1}{p^{\prime}}} A_{\infty}^{\exp }\left(w^{1-p^{\prime}}, Q\right)^{\frac{1}{p}} .
\end{aligned}
$$

Therefore, our result improves the above estimate as well.

Acknowledgements. The author would like to thank Prof. Tuomas P. Hytönen for suggesting this problem and for many helpful discussions which improve the quality of this paper.

\section{REFERENCES}

[1] T. Anderson, D. Cruz-Uribe and K. Moen, Logarithmic bump conditions for Calderón-Zygmund operators on spaces of homogeneous type, Publicacions Matematiques, 59(2015), 17-43. 2

[2] F. Bernicot, D. Frey and S. Petermichl, Sharp weighted norm estimates beyond Calderón-Zygmund theory, Anal. \& PDE, 9 (2016), No. 5, 1079-1113. 2, 3, 8

[3] Carme Cascante, Joaquin M. Ortega, and Igor E. Verbitsky. Nonlinear potentials and two weight trace inequalities for general dyadic and radial kernels. Indiana Univ. Math. J., 53(3):845-882, 2004. 7

[4] D. Cruz-Uribe and C. Pérez, Sharp two-weight, weak-type norm inequalities for singular integral operators, Math. Res. Lett. 6 (1999), no. 3-4, 417-427. 1 
[5] D. Cruz-Uribe and C. Pérez, Two-weight, weak-type norm inequalities for fractional integrals, Calderón- Zygmund operators and commutators, Indiana Univ. Math. J. 49 (2000), no. 2, 697-721. 1

[6] D. Cruz-Uribe, A. Reznikov and A. Volberg, Logarithmic bump conditions and the two-weight boundedness of Calderón-Zygmund operators, Adv. Math., 255(2014) 706-729. 1, 2

[7] T. Hänninen, Remark on dyadic pointwise domination and median oscillation decomposition, available at http://arxiv.org/abs/1502.05942. 2

[8] T. Hänninen, T. Hytönen and K. Li, Two-weight $L^{p}-L^{q}$ bounds for positive dyadic operators: unified approach to $p \leq q$ and $p>q$, Potential Anal.(2016), http://dx.doi.org/10.1007/s11118-016-9559-9 3, 6

[9] T. Hytönen, The sharp weighted bound for general Calderón-Zygmund operators, Ann. of Math., 175 (2012), 1473-1506. 1

[10] T. Hytönen and A. Kairema, Systems of dyadic cubes in a doubling metric space, Colloq. Math. 126 (2012), no. 1, 1-33. 2

[11] T. Hytönen and M. Lacey, The $A_{p}-A_{\infty}$ inequality for general Calderón-Zygmund operators, Indiana Univ. Math. J. 61 (2012), 2041-2052. 1, 4

[12] T. Hytönen and K. Li, Weak and strong $A_{p}-A_{\infty}$ estimates for square functions and related operators, preprint, available at http://arxiv.org/abs/1509.00273. 5

[13] T. Hytönen and C. Pérez, Sharp weighted bounds involving $A_{\infty}$, J. Anal. \& P.D.E., 6(2013), 777-818. 4

[14] M. Lacey, On the separated bumps conjecture for Calderón-Zygmund operators, Hokkaido Math. J., 45(2016), 223-242. 2, 13

[15] M. Lacey, E. Sawyer and I. Uriarte-Tuero, Two weight inequalities for discrete positiove operators, available at http://arxiv.org/abs/0911.3437. 3, 6

[16] M. Lacey and S. Spencer, On entropy bumps for Calderón-Zygmund operators. Concr. Oper. 2 (2015), 47-52. 4

[17] A. Lerner, A simple proof of the $A_{2}$ conjecture, Int. Math. Res. Not. 2012; doi: 10.1093/imrn/rns145. 1

[18] A. Lerner, On an estimate of Calderón-Zygmund operators by dyadic positive operators. J. Anal. Math. 121 (2013), 141-161. 15

[19] A. Lerner and K. Moen, Mixed $A_{p}-A_{\infty}$ estimates with one supremum, Studia Math., 219 (2013), 247-267. 15

[20] C. Pérez, Weighted norm inequalities for singular integral operators, J. London Math. Soc. 49(1994), no. 2, 296-308. 12

[21] H. Tanaka, A characterization of two-weight trace inequalities for positive dyadic operators in the upper triangle case, Potential Anal., 41(2):487-499, 2014. 3, 6

Department of Mathematics and Statistics, P.O.B. 68 (Gustaf Hällströmin KATU 2B), FI-00014 University of Helsinki, Finland

E-mail address: kangwei.li@helsinki.fi 